\title{
NOTE ON AN IRRATIONAL POWER SERIES
}

\section{H. DAVENPORT}

1. In several recent notes [1], [2], [3], [4] Mordell has investigated the series

$$
f(x)=\sum_{n=-\infty}^{\infty} \frac{a_{n} e(n \gamma)}{1-x e(n \alpha)},
$$

where $|x|<1$ and $\alpha, \gamma$ are real, and $e(\theta)=e^{2 \pi i \theta}$. If $\sum\left|a_{n}\right|$ converges, the series (1) obviously converges and has the alternative expression

$$
f(x)=\sum_{\nu=0}^{\infty} g(\nu \alpha+\gamma) x^{\nu}, \quad \text { where } g(t)=\sum_{-\infty}^{\infty} a_{n} e(n t) .
$$

Further, if $\alpha$ is irrational and $x=r e(k \alpha)$, where $k$ is an integer, it is easily proved that

$$
f(x) \sim \frac{a_{-k} e(-k \gamma)}{1-r} \text { as } r \rightarrow 1 \quad(0<r<1) .
$$

The same proof shows that if $x=r e(\theta)$, where $\theta$ is not congruent $(\bmod 1)$ to an integral multiple of $\alpha$, then $f(x)=o\left((1-r)^{-1}\right)$.

Now suppose that in (1), and in any later sums over $n$, the terms $n$ and $-n$ are taken together. Then Mordell has shown that there is another case, namely when

$$
a_{n}=\left\{\begin{array}{cc}
1 / n & \text { if } n \neq 0, \\
0 & \text { if } n=0,
\end{array}\right.
$$

in which the series (1) converges and equals (2), and in which the limit relation (3) holds. In this particular case, as is well known,

$$
g(t)=-2 \pi i\left(t-[t]-\frac{1}{2}\right)
$$

when $t$ is not an integer. It is also well known that in this particular case the partial sums $\sum_{-N}^{N} a_{n} e(n t)$ of the series for $g(t)$ are uniformly bounded.

The object of the present note is to give a simple and direct proof of these results, in a slightly more general form:

THEOREM. Suppose that $a_{n} \rightarrow 0$ as $n \rightarrow \pm \infty$, that $\sum_{-\infty}^{\infty} a_{n}$ converges, and that

Received by the editors November 2, 1964. 


$$
\sum_{-\infty}^{\infty}\left|a_{n}-a_{n+1}\right| \text { converges. }
$$

Suppose also that ${ }^{1}$ the partial sums $\sum_{-N}^{N} a_{n} e(n t)$ are uniformly bounded for all real $t$. Then for any real irrational $\alpha$ the two series for $f(x)$ in (1) and (2) converge and have the same sum, and the limit relation (3) holds.

In $\S 3$ we prove analogous results for the double series

$$
\sum_{-\infty}^{\infty} \sum_{-\infty}^{\infty} \frac{a_{m} a_{n} e(m \gamma+n \delta)}{1-x e(m \alpha+n \beta)}
$$

where $1, \alpha, \beta$ are linearly independent over the rationals. This answers, at least partially, a question raised in [3].

2. Proof of the theorem. We observe first that the convergence of the series for $g(t)$ in (2) follows by partial summation from the first hypotheses of the theorem, for any nonintegral $t$. Further, if

$$
g_{N}(t)=\sum_{|n|>N} a_{n} e(n t),
$$

partial summation gives

$$
\left|g_{N}(t)\right|<\delta(N)|| t||^{-1}
$$

where $\delta(N) \rightarrow 0$ as $N \rightarrow \infty$ and $\|t\|$ denotes the distance from $t$ to the nearest integer.

The final hypothesis of the theorem now implies that $g(t)$ is bounded, so the first series in (2) converges for $|x|<1$. Moreover we have

$$
\left|g_{N}(t)\right|<A
$$

where $A$ is independent of $t$ and $N$ (and similarly for $A_{1}, \cdots$, later).

The first series in (2) is

$$
\begin{array}{r}
\sum_{\nu=0}^{\infty} x^{\nu}\left(\sum_{-N}^{N} a_{n} e(n \nu \alpha+n \gamma)+g_{N}(\nu \alpha+\gamma)\right) \\
=\sum_{-N}^{N} \frac{a_{n} e(n \gamma)}{1-x e(n \alpha)}+\sum_{\nu=0}^{\infty} x^{\nu} g_{N}(\nu \alpha+\gamma) .
\end{array}
$$

Hence to establish the convergence of the series (1), and its equality

${ }^{1}$ This actually implies $a_{n} \rightarrow 0$, since it implies the convergence of $\sum\left|a_{n}\right|^{2}$. 
with (2), it suffices to prove that

$$
\sum_{\nu=0}^{\infty}|x| \nu\left|g_{N}(\nu \alpha+\gamma)\right| \rightarrow 0 \text { as } N \rightarrow \infty
$$

for any given $x$ with $|x|<1$. Here each single term $\rightarrow 0$ as $N \rightarrow \infty$, by (9) if $\nu \alpha+\gamma$ is not an integer, and by the convergence of $\sum a_{n}$ if $\nu \alpha+\gamma$ is an integer. Also, by (10),

$$
\sum_{\nu=h}^{\infty}|x|^{\nu}\left|g_{N}(\nu \alpha+\gamma)\right|<A \frac{|x|^{h}}{1-|x|},
$$

and this is arbitrarily small if $h$ is sufficiently large. Hence the result.

To prove the limit relation (3), we observe that the term $n=-k$ in (1) appears on the right of (3), and that any other single term is bounded as $r \rightarrow 1$, since $\alpha$ is irrational. Hence it suffices to prove that if $\epsilon>0$ is given then

$$
\left|\sum_{|n|>N} \frac{a_{n} e(n \gamma)}{1-r e(n \alpha+k \alpha)}\right|<\frac{\epsilon}{1-r}
$$

for any $N>N_{0}(\epsilon)$ and any $r$ with $r_{0}(\epsilon)<r<1$. The expression on the left is

$$
\begin{aligned}
& \leqq \sum_{\nu=0}^{\infty} r^{\nu}\left|g_{N}(\nu \alpha+\gamma)\right| \\
& <A_{1} \sum_{\nu=0}^{\infty} r^{\nu} \min \left(1, \delta(N)\|\nu \alpha+\gamma\|^{-1}\right),
\end{aligned}
$$

by (9) and (10).

We choose a large integer $q$ for which

$$
\alpha=\frac{a}{q}+\frac{\theta}{q^{2}}, \quad|\theta|<1 .
$$

Write $\nu=q u+v$ where $u \geqq 0$ and $0 \leqq v<q$. Then

$$
\begin{aligned}
\|\nu \alpha+\gamma\| & >\left\|\frac{a v}{q}+q \alpha u+\gamma\right\|-\frac{1}{q} \\
& >\left\|\frac{a v+w(u)}{q}\right\|-\frac{3}{2 q},
\end{aligned}
$$

where $w(u)$ is the integer nearest to $q^{2} \alpha u+q \gamma$. For given $u$, we define $v^{\prime}$ (as a function of $v$ ) to be the absolutely least residue of $a v+w(u)$ 
modulo $q$. In the sum (12) we take 1 in the minimum if $\left|v^{\prime}\right| \leqq 2$, and find that the expression is

$$
\begin{aligned}
& <A_{1} \sum_{u=0}^{\infty} r^{q u}\left(5+2 \sum_{v^{\prime}=3}^{q / 2} \frac{\delta(N) q}{v^{\prime}-2}\right) \\
& <A_{2}(1+\delta(N) q \log q) \frac{1}{1-r^{q}} .
\end{aligned}
$$

Hence (11) will hold provided that

$$
\Lambda_{2}(1+\delta(N) q \log q)<\epsilon\left(1+r+r^{2}+\cdots+r^{q-1}\right) .
$$

The right-hand side is greater than $\frac{1}{2} \epsilon q$ if $r^{q}>\frac{1}{2}$. We first choose $q$ so that $A_{2}<\frac{1}{4} \epsilon q$, then choose $N_{0}$ so that $A_{2} \delta(N) \log q<\frac{1}{4} \epsilon$ for $N>N_{0}$, then choose $r_{0}$ so that $r_{0}^{q}>\frac{1}{2}$. Then (11) holds, and this completes the proof of (3).

3. The double series (7). The proofs of the analogous results, under the hypotheses of the theorem, present no additional difficulty. We have

$$
\begin{aligned}
\sum_{\nu=0}^{\infty} x^{\nu} g(\nu \alpha & +\gamma) g(\nu \beta+\delta) \\
& =\sum_{m=-M}^{M} \sum_{n=-N}^{N} \frac{a_{m} a_{n} e(m \gamma+n \delta)}{1-x e(m \alpha+n \beta)}+S_{1}+S_{2}-S_{3},
\end{aligned}
$$

where

$$
S_{1}=\sum_{\nu=0}^{\infty} x^{\nu} g(\nu \alpha+\gamma) g_{N}(\nu \beta+\delta)
$$

and $S_{2}$ is a similar sum, and

$$
S_{3}=\sum_{\nu=0}^{\infty} x^{\nu} g_{M}(\nu \alpha+\gamma) g_{N}(\nu \beta+\delta) .
$$

Since $g(t)$ is bounded and $g_{N}(t)$ is uniformly bounded, and (as we have already proved)

$$
\sum_{\nu=0}^{\infty}|x| \nu\left|g_{N}(\nu \alpha+\gamma)\right| \rightarrow 0 \text { as } N \rightarrow \infty,
$$

it follows that the double series (7) converges and has the sum $\sum x^{\prime} g(\nu \alpha+\gamma) g(\nu \beta+\delta)$. 
As for the analogue of the limit relation, it suffices to prove that

$$
\left|\sum_{\max (|m|,|n|)>N} \frac{a_{m} a_{n} e(m \gamma+n \delta)}{1-r e(m \alpha+n \beta+k \alpha+l \beta)}\right|<\frac{\epsilon}{1-r}
$$

for $N>N_{0}(\epsilon)$ and $r_{0}(\epsilon)<r<1$. The double sum here can be expressed again as $S_{1}+S_{2}-S_{3}$, where $x$ is now replaced by $r e(k \alpha+l \beta)$, and now $M=N$. Since $g(t)$ is bounded and $g_{N}(t)$ is uniformly bounded, the desired estimate follows from what was proved in $\$ 2$.

We can obviously replace $a_{n}$ by $b_{n}$ in (7), provided the sequence $b_{n}$ satisfies the same conditions as $a_{n}$.

\section{REFERENCES}

1. L. J. Mordell, The series $\sum a_{n} /\left(1-x e^{2 n \pi i a}\right)$, J. London Math. Soc. 38 (1963), 111-116.

2. - Irrational power series. I, Proc. Amer. Math. Soc. 12 (1961), 522-526.

3. - Irrational power series. II, Acta Arith. 11 (1965), 181-188.

4. - Irrational power series. III, Proc. Amer. Math. Soc. 16 (1965), 819-821.

Trinity College, Cambridge, England 